\title{
ESCRIBED AND INSCRIBED SIMPLICES AND BEVAN POINT THEOREM ON $n$-DIMENSIONAL EUCLIDEAN SPACE WITH
} $n \geqq 3$

\author{
KENZI SATÔ
}

(Communicated by H. Hilmi HACISALIHOĞLU)

\begin{abstract}
Escribed and inscribed triangles for a triangle on the plane is generalized to a simplex on the $n$-dimensional Euclidean space with $n \geqq 3$. As an application, we can get Bevan Point Theorem for a simplex on the $n$-dimensional Euclidean space.
\end{abstract}

\section{INTRODUCTION.}

For a triangle on the plane, three perpendiculars from vertices to the opposite edges are concurrent and their common point is called the orthocenter of the triangle. Moreover, the common point is consistent with the incenter of the inscribed triangle, whose vetices are feet of the perpendiculars above. Conversely, the incenter of a triangle is consitent with the orthocenter of the escribed triangle, whose vetices are excenters. In this paper we generalize it to a simplex on higher dimensional Euclidean space, however, for higher dimensional case, the perpendiculars from vertices to the opposite faces are not concurrent in general, so there exist several ways of generalization. We choose one of these ways of generalization, because in the way we can calculate the escribed and inscribed simplices explicitly and we can generalize the following theorem to a simplex on higher dimensional Euclidean space:

Theorem 1.1. (Bevan point theorem) [2] Let $\Delta^{2}\left(\mathbf{q}_{0}, \mathbf{q}_{1}, \mathbf{q}_{2}\right)$ be a triangle on the plane $\mathbb{R}^{2}$ with vertices $\mathbf{q}_{0}, \mathbf{q}_{1}$, and, $\mathbf{q}_{2}$, and $\Delta^{2}\left(\mathbf{q}_{0}^{\mathrm{E}}, \mathbf{q}_{1}^{\mathrm{E}}, \mathbf{q}_{2}^{\mathrm{E}}\right)$ the triangle with vertices $\mathbf{q}_{0}^{\mathrm{E}}, \mathbf{q}_{1}^{\mathrm{E}}$, and $\mathbf{q}_{2}^{\mathrm{E}}$, which are excenters of $\Delta^{2}\left(\mathbf{q}_{0}, \mathbf{q}_{1}, \mathbf{q}_{2}\right)$. Then, the vector $\mathbf{c}^{\mathrm{E}}-\mathbf{q}_{i}^{\mathrm{E}}$ is perpendicular to the edge $\Delta^{1}\left(\mathbf{q}_{k}, \mathbf{q}_{\ell}\right)$ for distinct $i, k, \ell=0,1,2$, and we have

$$
\mathbf{c}^{\mathrm{E}}=2 \mathbf{c}-\mathbf{i}, \quad R^{\mathrm{E}}=2 R,
$$

where $\mathbf{i}, \mathbf{c}$, and $R$ are the incenter, the circumcenter, and the circumradius of $\Delta^{2}\left(\mathbf{q}_{0}, \mathbf{q}_{1}, \mathbf{q}_{2}\right)$, and $\mathbf{c}^{\mathrm{E}}$ and $R^{\mathrm{E}}$ are the circumcenter and the circumradius of $\Delta^{2}\left(\mathbf{q}_{0}^{\mathrm{E}}, \mathbf{q}_{1}^{\mathrm{E}}, \mathbf{q}_{2}^{\mathrm{E}}\right)$, respectively.

Date: Received: October 13, 2014 and Accepted: September 9, 2015.

2010 Mathematics Subject Classification. Primary 51M04; Secondary 51M05.

Key words and phrases. Bevan point theorem, circumcenter, circumradius, escribed simplex, incenter, inradius, inscribed simplex, simplex. 


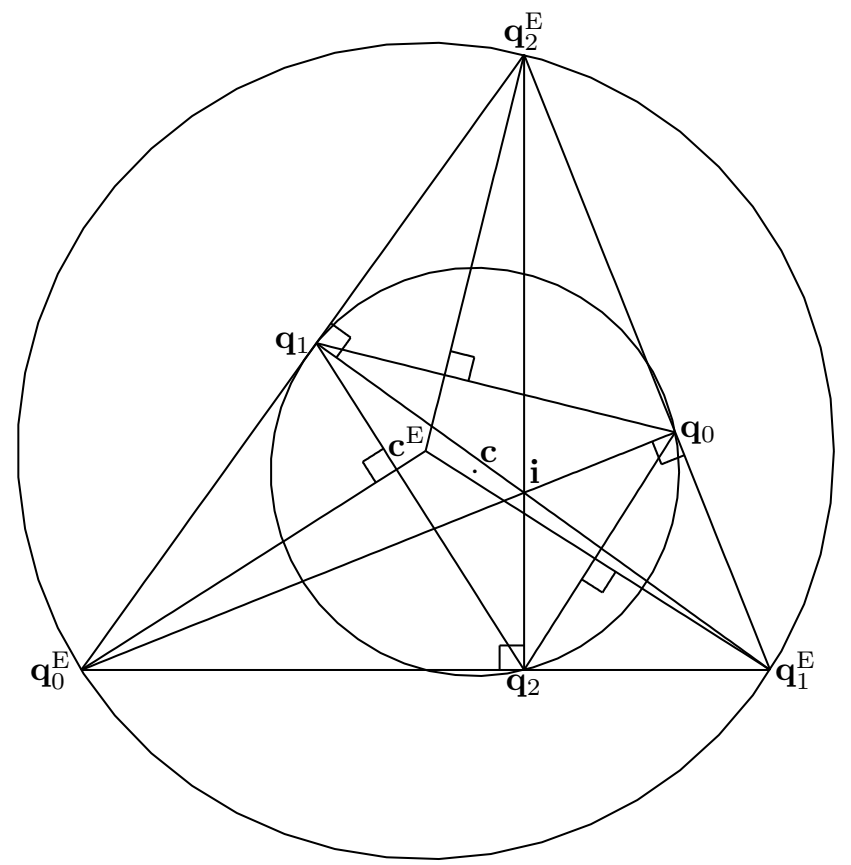

There exist three ways of generalization of the escribed and the inscribed triangles of a triangle on $\mathbb{R}^{2}$ to the escribed and the inscribed simplices of a simplex on $\mathbb{R}^{n}$ for $n \geqq 3$.

(A) For a simplex $\Delta^{n}\left(\mathbf{q}_{0}, \ldots, \mathbf{q}_{n}\right)$ on $\mathbb{R}^{n}$, the inscribed simplex is the simplex such that the $i$-th vertex of it is the foot of the perpendicular from the vertex $\mathbf{q}_{i}$ to the opposite face $\Delta^{n-1}\left(\mathbf{q}_{0}, \ldots \widehat{\mathbf{q}_{i}} \ldots, \mathbf{q}_{n}\right)$, where the circumflex indicates that the term below it has been omitted. In overview, the escribed simplex is the image of the inverse mapping of the mapping from simplices to their inscribed simplices.

(B) For a simplex $\Delta^{n}\left(\mathbf{q}_{0}, \ldots, \mathbf{q}_{n}\right)$ on $\mathbb{R}^{n}$, the escribed simplex is the simplex such that the opposite face of the $i$-th vertex of it is including $\mathbf{q}_{i}$ and perpendicular to the straight line passing through $\mathbf{i}$ and $\mathbf{q}_{i}$. In overview, the inscribed simplex is the image of the inverse mapping of the mapping from simplices to their escribed simplices.

(C) For a simplex $\Delta^{n}\left(\mathbf{q}_{0}, \ldots, \mathbf{q}_{n}\right)$ on $\mathbb{R}^{n}$, the escribed simplex is the simplex such that the $i$-th vertex of it is on the straight line passing through $\mathbf{i}$ and $\mathbf{q}_{i}$ and its opposite face is including $\mathbf{q}_{i}$. In overview, the inscribed simplex is the image of the inverse mapping of the mapping from simplices to their escribed simplices. 
(A)

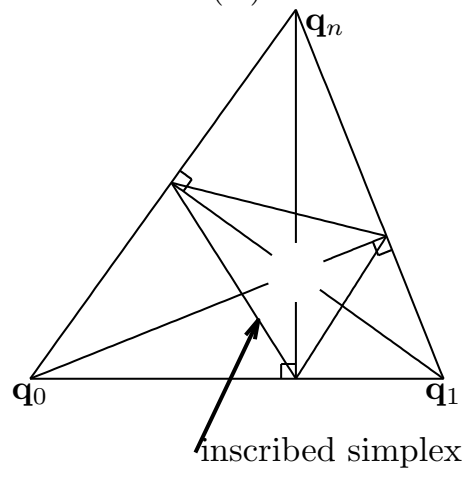

(B)

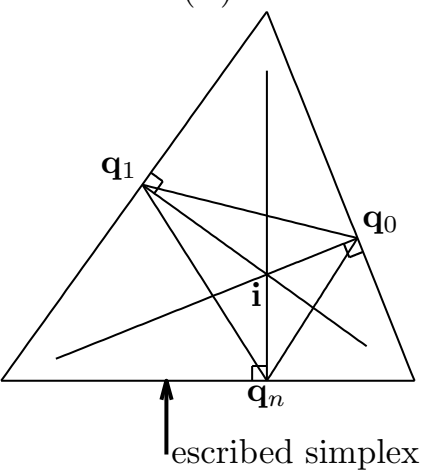

(C)

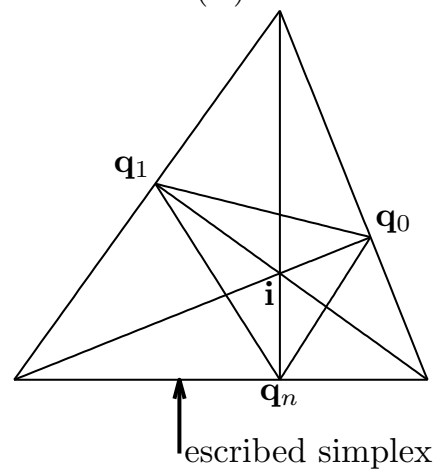

In this paper we use (B):

Definition 1.1. For a simplex

$$
\Delta^{n}\left(\mathbf{q}_{0}, \ldots, \mathbf{q}_{n}\right)=\left\{\sum_{j=0}^{n} x_{j} \mathbf{q}_{j} \in \mathbb{R}^{n}: \sum_{j=0}^{n} x_{j}=1, x_{j} \geqq 0 \text { for each } j=0, \ldots, n\right\},
$$

the escribed simplex $\Delta^{n}\left(\mathbf{q}_{0}^{\mathrm{E}}, \ldots, \mathbf{q}_{n}^{\mathrm{E}}\right)$ is defined by the simplex with $(n-1)$-dimensional face $\Delta^{n-1}\left(\mathbf{q}_{0}^{\mathrm{E}}, \ldots \widehat{\mathbf{q}_{i}^{\mathrm{E}}} \ldots, \mathbf{q}_{n}^{\mathrm{E}}\right)$ which is including $\mathbf{q}_{i}$ and perpendicular to the vector $\mathbf{i}-\mathbf{q}_{i}$. For a simplex $\Delta^{n}\left(\mathbf{q}_{0}, \ldots, \mathbf{q}_{n}\right)$ whose circumcenter is an interior of $\Delta^{n}\left(\mathbf{q}_{0}, \ldots, \mathbf{q}_{n}\right)$, the inscribed simplex is defined by the simplex whose escribed simplex is $\Delta^{n}\left(\mathbf{q}_{0}, \ldots, \mathbf{q}_{n}\right)$ (notice that the inscribed simplex is uniquely determined, see Theorem 4.1).

The reason why we use the definition above is that we can calculate the escribed and inscribed simplices explicitly and we can get the following theorem:

Theorem 1.2. (generalized Bevan point theorem) Let $\Delta^{n}\left(\mathbf{q}_{0}, \ldots, \mathbf{q}_{n}\right)$ be a simplex on $\mathbb{R}^{n}$ and $\Delta^{n}\left(\mathbf{q}_{0}^{\mathrm{E}}, \ldots, \mathbf{q}_{n}^{\mathrm{E}}\right)$ its escribed simplex. Then, the vector $\mathbf{c}^{\mathrm{E}}-\mathbf{q}_{i}^{\mathrm{E}}$ is perpendicular to $\Delta^{n-1}\left(\mathbf{q}_{0}, \ldots \widehat{\mathbf{q}}_{i} \ldots, \mathbf{q}_{n}\right)$ for $i=0, \ldots, n$, and we have

$$
\mathbf{c}^{\mathrm{E}}=2 \mathbf{c}-\mathbf{i}, \quad R^{\mathrm{E}}=\frac{R^{2}-|\mathbf{c}-\mathbf{i}|^{2}}{r},
$$

where $\mathbf{i}, \mathbf{c}, r$, and $R$ are the incenter, the circumcenter, the inradius, and the circumradius of $\Delta^{n}\left(\mathbf{q}_{0}, \ldots, \mathbf{q}_{n}\right)$, and $\mathbf{c}^{\mathrm{E}}$ and $R^{\mathrm{E}}$ are the circumcenter and the circumradius of $\Delta^{n}\left(\mathbf{q}_{0}^{\mathrm{E}}, \ldots, \mathbf{q}_{n}^{\mathrm{E}}\right)$, respectively.

Remark 1.1. For $n=2$, the equation $\frac{R^{2}-|\mathbf{c}-\mathbf{i}|^{2}}{r}=2 R$ holds. This is a natural consequence from Theorems 1.1 and 1.2, but we also have it by direct calculation. See Remark 2.3.

\section{Preliminaries.}

For a simplex $\Delta^{n}\left(\mathbf{q}_{0}, \ldots, \mathbf{q}_{n}\right)$ on $\mathbb{R}^{n}$, the family of vectors $\left\{\mathbf{q}_{0}-\mathbf{q}_{n}, \ldots, \mathbf{q}_{n-1}-\right.$ $\left.\mathbf{q}_{n}\right\}$ is linearly independent, so it is a basis of $\mathbb{R}^{n}$. Let $\left\{\mathbf{t}_{0}, \ldots, \mathbf{t}_{n-1}\right\}$ be the dual basis of it, i.e., $\left(\mathbf{q}_{i}-\mathbf{q}_{n}\right) \cdot \mathbf{t}_{k}=\delta_{i k}$ for $i, k=0, \ldots, n-1$, and let $\mathbf{t}_{n}=-\sum_{k=0}^{n-1} \mathbf{t}_{k}$. Then we have the following proposition and lemma.

Proposition 2.1. For each $j=0, \ldots, n$, the dual basis of $\left\{\mathbf{q}_{0}-\mathbf{q}_{j}, \ldots \widehat{\mathbf{q}_{j}-\mathbf{q}_{j}} \ldots, \mathbf{q}_{n}-\right.$ $\left.\mathbf{q}_{j}\right\}$ is $\left\{\mathbf{t}_{0}, \ldots \widehat{\mathbf{t}_{j}} \ldots, \mathbf{t}_{n}\right\}$, i.e., $\left(\mathbf{q}_{i}-\mathbf{q}_{j}\right) . \mathbf{t}_{k}=\delta_{i k}-\delta_{j k}$ for $i, k=0, \ldots, n$. 
Remark 2.1. Let

$$
\tilde{V}=(-1)^{n-j} \operatorname{det}\left(\mathbf{q}_{0}-\mathbf{q}_{j}, \ldots \widehat{\mathbf{q}_{j}-\mathbf{q}_{j}} \ldots, \mathbf{q}_{n}-\mathbf{q}_{j}\right),
$$

which does not depend on the index $j=0, \ldots, n$, where $\left(\mathbf{v}_{0}, \ldots, \mathbf{v}_{n-1}\right)$ is the matrix that has $\mathbf{v}_{0}, \ldots, \mathbf{v}_{n-1}$ as column vectors. Then the signed volume of the simplex $\Delta^{n}\left(\mathbf{q}_{0}, \ldots, \mathbf{q}_{n}\right)$ is $\tilde{V} / n$ ! and

$$
\tilde{V}^{2}=\operatorname{det}\left(\begin{array}{ccc}
\left(\mathbf{q}_{0}-\mathbf{q}_{n}\right) \cdot\left(\mathbf{q}_{0}-\mathbf{q}_{n}\right) & \cdots & \left(\mathbf{q}_{0}-\mathbf{q}_{n}\right) \cdot\left(\mathbf{q}_{n-1}-\mathbf{q}_{n}\right) \\
\vdots & & \vdots \\
\left(\mathbf{q}_{n-1}-\mathbf{q}_{n}\right) \cdot\left(\mathbf{q}_{0}-\mathbf{q}_{n}\right) & \cdots & \left(\mathbf{q}_{n-1}-\mathbf{q}_{n}\right) \cdot\left(\mathbf{q}_{n-1}-\mathbf{q}_{n}\right)
\end{array}\right)
$$

holds. For $i=0, \ldots, n$, let

$$
\begin{aligned}
\tilde{\mathbf{t}}_{i} & =(-1)^{i-k}\left\langle\left\langle\mathbf{q}_{0}-\mathbf{q}_{k}, \ldots \widehat{\mathbf{q}_{k}-\mathbf{q}_{k}} \ldots \widehat{\mathbf{q}_{i}-\mathbf{q}_{k}} \ldots, \mathbf{q}_{n}-\mathbf{q}_{k}\right\rangle\right\rangle= \\
& =(-1)^{\ell-i-1}\left\langle\left\langle\mathbf{q}_{0}-\mathbf{q}_{\ell}, \ldots \widehat{\mathbf{q}_{i}-\mathbf{q}_{\ell}} \ldots \widehat{\mathbf{q}_{\ell}-\mathbf{q}_{\ell}} \ldots, \mathbf{q}_{n}-\mathbf{q}_{\ell}\right\rangle\right\rangle
\end{aligned}
$$

which does not depend on $k=0, \ldots, i-1$, and $\ell=i+1, \ldots, n$, where $\left\langle\left\langle\mathbf{v}_{0}, \ldots, \mathbf{v}_{n-2}\right\rangle\right\rangle$ is the unique vector such that

$$
\left\langle\left\langle\mathbf{v}_{0}, \ldots, \mathbf{v}_{n-2}\right\rangle\right\rangle \cdot \mathbf{v}_{n-1}=\operatorname{det}\left(\mathbf{v}_{0}, \ldots, \mathbf{v}_{n-2}, \mathbf{v}_{n-1}\right),
$$

for all $\mathbf{v}_{n-1} \in \mathbb{R}^{n}$. Then, $\tilde{\mathbf{t}}_{i}$ is perpendicular to the face $\Delta^{n-1}\left(\mathbf{q}_{0}, \ldots \widehat{\mathbf{q}_{i}} \ldots, \mathbf{q}_{n}\right)$, the volume of $\Delta^{n-1}\left(\mathbf{q}_{0}, \ldots \widehat{\mathbf{q}_{i}} \ldots, \mathbf{q}_{n}\right)$ is $\left|\tilde{\mathbf{t}}_{i}\right| /(n-1)$ !, and $\mathbf{t}_{i}=\tilde{\mathbf{t}}_{i} / \tilde{V}$ holds. See [4].

Lemma 2.1. Let $\mathbf{x}=\sum_{j=0}^{n} x_{j} \mathbf{q}_{j}$ be a point in $\mathbb{R}^{n}$ with $\sum_{j=0}^{n} x_{j}=1$. Then, the foot of the perpendicular from $\mathbf{x}$ to the face $\Delta^{n-1}\left(\mathbf{q}_{0}, \ldots \widehat{\mathbf{q}_{k}} \ldots, \mathbf{q}_{n}\right)$ is $\mathbf{x}-\frac{x_{k} \mathbf{t}_{k}}{\left|\mathbf{t}_{k}\right|^{2}}$. Especially, the foot of the perpendicular from the vertex $\mathbf{q}_{k}$ to the opposite face $\Delta^{n-1}\left(\mathbf{q}_{0}, \ldots \widehat{\mathbf{q}_{k}} \ldots, \mathbf{q}_{n}\right)$ is $\mathbf{q}_{k}-\frac{\mathbf{t}_{k}}{\left|\mathbf{t}_{k}\right|^{2}}$.

Proof. The vector $\mathbf{t}_{k}$ is perpendicular to $\Delta^{n-1}\left(\mathbf{q}_{0}, \ldots \widehat{\mathbf{q}_{k}} \ldots, \mathbf{q}_{n}\right)$, so it is enough to show that the point $\mathbf{x}-\frac{x_{k} \mathbf{t}_{k}}{\left|\mathbf{t}_{k}\right|^{2}}$ is in the affined space spanned by $\Delta^{n-1}\left(\mathbf{q}_{0}, \ldots \widehat{\mathbf{q}_{k}} \ldots, \mathbf{q}_{n}\right)$. In particular, we have

$$
\begin{aligned}
\mathbf{x}-\frac{x_{k} \mathbf{t}_{k}}{\left|\mathbf{t}_{k}\right|^{2}} & =\mathbf{x}-\frac{x_{k}}{\left|\mathbf{t}_{k}\right|^{2}} \sum_{j=0}^{\substack{j \neq k \\
n}}\left(\mathbf{t}_{k} \cdot \mathbf{t}_{j}\right)\left(\mathbf{q}_{j}-\mathbf{q}_{k}\right)= \\
& =\mathbf{x}-\frac{x_{k}}{\left|\mathbf{t}_{k}\right|^{2}}\left(\sum_{j=0}^{\substack{j \neq k \\
n}}\left(\mathbf{t}_{k} \cdot \mathbf{t}_{j}\right) \mathbf{q}_{j}+\left|\mathbf{t}_{k}\right|^{2} \mathbf{q}_{k}\right)=\sum_{j=0}^{\substack{j \neq k \\
n}}\left(x_{j}-\frac{x_{k} \mathbf{t}_{k} \cdot \mathbf{t}_{j}}{\left|\mathbf{t}_{k}\right|^{2}}\right) \mathbf{q}_{j}
\end{aligned}
$$

where the first equality is from Proposition 2.1 and the second equality is from $\sum_{j=0}^{n} \mathbf{t}_{j}=\mathbf{0}$.

Remark 2.2. So the height of $\mathbf{x}$ from $\Delta^{n-1}\left(\mathbf{q}_{0}, \ldots \widehat{\mathbf{q}_{k}} \ldots, \mathbf{q}_{n}\right)$ is $\left|\mathbf{x}-\left(\mathbf{x}-\frac{x_{k} \mathbf{t}_{k}}{\left|\mathbf{t}_{k}\right|^{2}}\right)\right|=$ $\frac{\left|x_{k}\right|}{\left|\mathbf{t}_{k}\right|}$. Especially, the height of $\mathbf{q}_{k}$ from the opposite face is $\frac{1}{\left|\mathbf{t}_{k}\right|}$. 

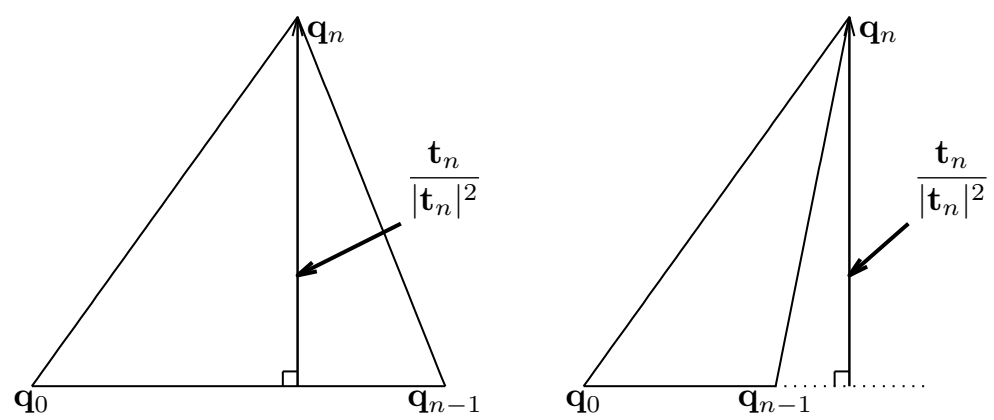

We can represent centers and radiuses of the insphere and the circumsphere of a simplex.

Lemma 2.2. The incenter $\mathbf{i}$, the inradius $r$, the circumcenter $\mathbf{c}$, and the circumradius $R$ of $\Delta^{n}\left(\mathbf{q}_{0}, \ldots, \mathbf{q}_{n}\right)$ can be represented by the following:

$$
\begin{gathered}
\mathbf{i}=r \sum_{j=0}^{n}\left|\mathbf{t}_{j}\right| \mathbf{q}_{j}, \quad r=\frac{1}{\sum_{j=0}^{n}\left|\mathbf{t}_{j}\right|}, \\
\mathbf{c}=\mathbf{q}_{i}+\frac{1}{2} \sum_{j=0}^{n} d_{i j}^{2} \mathbf{t}_{j}, \quad R=\left|\frac{1}{2} \sum_{j=0}^{n} d_{i j}^{2} \mathbf{t}_{j}\right| \quad \text { for } i=0, \ldots, n,
\end{gathered}
$$

where $d_{i j}=\left|\mathbf{q}_{i}-\mathbf{q}_{j}\right|$.

Proof. For $\mathbf{i}=\sum_{j=0}^{n} x_{j} \mathbf{q}_{j}$ with $\sum_{j=0}^{n} x_{j}=1$, notice that $x_{j}>0$ for each $j=0, \ldots$, $n$. The height $\frac{x_{k}}{\left|\mathbf{t}_{k}\right|}$ of $\mathbf{i}$ from $\Delta^{n-1}\left(\mathbf{q}_{0}, \ldots \widehat{\mathbf{q}_{k}} \ldots, \mathbf{q}_{n}\right)$ does not depend on the index $k=0, \ldots, n$, because the height is equal to the inradius $r$. So $x_{k}=\frac{\left|\mathbf{t}_{k}\right|}{\sum_{j=0}^{n}\left|\mathbf{t}_{j}\right|}$ and $r=\frac{x_{k}}{\left|\mathbf{t}_{k}\right|}=\frac{1}{\sum_{j=0}^{n}\left|\mathbf{t}_{j}\right|}$. For the circumcenter and the circumradius, it is enough to notice that the result of the following calculation does not depend on $k$ :

$$
\begin{aligned}
& \left|\mathbf{q}_{k}-\left(\mathbf{q}_{i}+\frac{1}{2} \sum_{j=0}^{n} d_{i j}^{2} \mathbf{t}_{j}\right)\right|^{2}=\left|\left(\mathbf{q}_{k}-\mathbf{q}_{i}\right)-\frac{1}{2} \sum_{j=0}^{n} d_{i j}^{2} \mathbf{t}_{j}\right|^{2}= \\
& =d_{i k}^{2}-\sum_{j=0}^{n} d_{i j}^{2}\left(\delta_{k j}-\delta_{i j}\right)+\left|\frac{1}{2} \sum_{j=0}^{n} d_{i j}^{2} \mathbf{t}_{j}\right|^{2}=\left|\frac{1}{2} \sum_{j=0}^{n} d_{i j}^{2} \mathbf{t}_{j}\right|^{2} .
\end{aligned}
$$

The following lemma is useful.

Lemma 2.3. The following equations hold:

$$
\begin{gathered}
\mathbf{x}-\mathbf{c}=-\frac{1}{2} \sum_{i=0}^{n}\left|\mathbf{x}-\mathbf{q}_{i}\right|^{2} \mathbf{t}_{i}, \\
c_{i}=\frac{1}{2} \sum_{k=0}^{n} d_{j k}^{2} \mathbf{t}_{k} \cdot \mathbf{t}_{i}+\delta_{i j} \quad \text { for } i, j=0, \ldots, n,
\end{gathered}
$$




$$
R^{2}=\frac{1}{2} \sum_{j=0}^{n} d_{i j}^{2} c_{j} \quad \text { for } i=0, \ldots, n, \quad R^{2}=\frac{1}{2} \sum_{i=0}^{n} \sum_{j=0}^{n} d_{i j}^{2} c_{i} c_{j}
$$

$$
\begin{gathered}
(\mathbf{x}-\mathbf{c}) \cdot(\mathbf{y}-\mathbf{c})=-\frac{1}{2} \sum_{i=0}^{n} \sum_{j=0}^{n} d_{i j}^{2}\left(x_{i}-c_{i}\right)\left(y_{j}-c_{j}\right)=R^{2}-\frac{1}{2} \sum_{i=0}^{n} \sum_{j=0}^{n} d_{i j}^{2} x_{i} y_{j}, \\
|\mathbf{x}-\mathbf{y}|^{2}=-\frac{1}{2} \sum_{i=0}^{n} \sum_{j=0}^{n} d_{i j}^{2}\left(x_{i}-y_{i}\right)\left(x_{j}-y_{j}\right) \\
\sum_{j=0}^{n}\left|\mathbf{t}_{j}\right|\left|\mathbf{q}_{j}-\mathbf{i}\right|^{2}=\frac{R^{2}-|\mathbf{c}-\mathbf{i}|^{2}}{r}
\end{gathered}
$$

for arbitrary $\mathbf{x}=\sum_{i=0}^{n} x_{i} \mathbf{q}_{i}$ and $\mathbf{y}=\sum_{i=0}^{n} y_{i} \mathbf{q}_{i}$ in $\mathbb{R}^{n}$ where $\mathbf{c}=\sum_{i=0}^{n} c_{i} \mathbf{q}_{i}$ with $\sum_{i=0}^{n} x_{i}=\sum_{i=0}^{n} y_{i}=\sum_{i=0}^{n} c_{i}=1$.

Proof. We have all equations by the following direct calculations. For (2.1):

$$
\begin{aligned}
\mathbf{x}-\mathbf{c} & =\mathbf{0}+\left(\mathbf{x}-\mathbf{q}_{n}\right)-\left(\mathbf{c}-\mathbf{q}_{n}\right)= \\
& =\sum_{i=0}^{n}\left(-\frac{1}{2}\left|\mathbf{x}-\mathbf{q}_{n}\right|^{2}+\left(\mathbf{x}-\mathbf{q}_{n}\right) \cdot\left(\mathbf{q}_{i}-\mathbf{q}_{n}\right)-\frac{1}{2} d_{i n}^{2}\right) \mathbf{t}_{i}= \\
& =-\frac{1}{2} \sum_{i=0}^{n}\left|\left(\mathbf{x}-\mathbf{q}_{n}\right)-\left(\mathbf{q}_{i}-\mathbf{q}_{n}\right)\right|^{2} \mathbf{t}_{i}=-\frac{1}{2} \sum_{i=0}^{n}\left|\mathbf{x}-\mathbf{q}_{i}\right|^{2} \mathbf{t}_{i},
\end{aligned}
$$

where the second equality is from $\sum_{j=0}^{n} \mathbf{t}_{j}=\mathbf{0}$, Proposition 2.1, and Lemma 2.2. For (2.2):

$$
\begin{aligned}
\frac{1}{2} \sum_{k=0}^{n} d_{j k}^{2} \mathbf{t}_{k} \cdot \mathbf{t}_{i} & =\left(\mathbf{c}-\mathbf{q}_{j}\right) \cdot \mathbf{t}_{i}=\sum_{k=0}^{n} c_{k}\left(\mathbf{q}_{k}-\mathbf{q}_{j}\right) \cdot \mathbf{t}_{i}= \\
& =\sum_{k=0}^{n} c_{k}\left(\delta_{i k}-\delta_{i j}\right)=c_{i}-\delta_{i j} .
\end{aligned}
$$

For (2.3):

$$
\begin{aligned}
\frac{1}{2} \sum_{j=0}^{n} d_{i j}^{2} c_{j} & =\frac{1}{2} \sum_{j=0}^{n} d_{i j}^{2}\left(c_{j}-\delta_{j n}\right)+\frac{1}{2} d_{i n}^{2}=\frac{1}{2} \sum_{j=0}^{n} d_{i j}^{2}\left(\frac{1}{2} \sum_{k=0}^{n} d_{k n}^{2} \mathbf{t}_{k} \cdot \mathbf{t}_{j}\right)+\frac{1}{2} d_{i n}^{2}= \\
& =\frac{1}{2} \sum_{k=0}^{n} d_{k n}^{2} \mathbf{t}_{k} \cdot \frac{1}{2} \sum_{j=0}^{n} d_{i j}^{2} \mathbf{t}_{j}+\frac{1}{2} \sum_{j=0}^{n} d_{i j}^{2}\left(\delta_{j n}-\delta_{i j}\right)= \\
& =\left(\frac{1}{2} \sum_{k=0}^{n} d_{k n}^{2} \mathbf{t}_{k}+\left(\mathbf{q}_{n}-\mathbf{q}_{i}\right)\right) \cdot \frac{1}{2} \sum_{j=0}^{n} d_{i j}^{2} \mathbf{t}_{j}= \\
& =\left(\left(\mathbf{c}-\mathbf{q}_{n}\right)+\left(\mathbf{q}_{n}-\mathbf{q}_{i}\right)\right) \cdot\left(\mathbf{c}-\mathbf{q}_{i}\right)=R^{2},
\end{aligned}
$$

and

$$
\frac{1}{2} \sum_{i=0}^{n} \sum_{j=0}^{n} d_{i j}^{2} c_{i} c_{j}=\sum_{i=0}^{n}\left(\frac{1}{2} \sum_{j=0}^{n} d_{i j}^{2} c_{j}\right) c_{i}=\sum_{i=0}^{n} R^{2} c_{i}=R^{2}
$$


For (2.4):

$$
\begin{aligned}
& (\mathbf{x}-\mathbf{c}) \cdot(\mathbf{y}-\mathbf{c})=\sum_{i=0}^{n} x_{i}^{\prime}\left(\mathbf{q}_{i}-\mathbf{q}_{n}\right) \cdot \sum_{j=0}^{n} y_{j}^{\prime}\left(\mathbf{q}_{j}-\mathbf{q}_{n}\right)=\sum_{i=0}^{n} \sum_{j=0}^{n} \frac{d_{i n}^{2}+d_{j n}^{2}-d_{i j}^{2}}{2} x_{i}^{\prime} y_{j}^{\prime}= \\
& =\frac{1}{2}\left(\sum_{i=0}^{n} d_{i n}^{2} x_{i}^{\prime}\left(\sum_{j=0}^{n} y_{j}^{\prime}\right)+\left(\sum_{i=0}^{n} x_{i}^{\prime}\right) \sum_{j=0}^{n} d_{j n}^{2} y_{j}^{\prime}-\sum_{i=0}^{n} \sum_{j=0}^{n} d_{i j}^{2} x_{i}^{\prime} y_{j}^{\prime}\right)=-\frac{1}{2} \sum_{i=0}^{n} \sum_{j=0}^{n} d_{i j}^{2} x_{i}^{\prime} y_{j}^{\prime},
\end{aligned}
$$

where the last equality is from $\sum_{i=0}^{n} x_{i}^{\prime}=\sum_{i=0}^{n} y_{i}^{\prime}=0$ with $x_{i}^{\prime}=x_{i}-c_{i}$ and $y_{i}^{\prime}=y_{i}-c_{i}$, and

$$
\begin{aligned}
& R^{2}-\frac{1}{2} \sum_{i=0}^{n} \sum_{j=0}^{n} d_{i j}^{2} x_{i} y_{j}= \\
& =R^{2}-\frac{1}{2}\left(\sum_{i=0}^{n} \sum_{j=0}^{n} d_{i j}^{2} c_{i} c_{j}+\sum_{i=0}^{n}\left(\sum_{j=0}^{n} d_{i j}^{2} c_{j}\right) x_{i}^{\prime}+\sum_{j=0}^{n}\left(\sum_{i=0}^{n} d_{i j}^{2} c_{i}\right) y_{j}^{\prime}+\sum_{i=0}^{n} \sum_{j=0}^{n} d_{i j}^{2} x_{i}^{\prime} y_{j}^{\prime}\right)= \\
& =R^{2}-\frac{1}{2}\left(2 R^{2}+\sum_{i=0}^{n} 2 R^{2} x_{i}^{\prime}+\sum_{j=0}^{n} 2 R^{2} y_{j}^{\prime}+\sum_{i=0}^{n} \sum_{j=0}^{n} d_{i j}^{2} x_{i}^{\prime} y_{j}^{\prime}\right)=-\frac{1}{2} \sum_{i=0}^{n} \sum_{j=0}^{n} d_{i j}^{2} x_{i}^{\prime} y_{j}^{\prime},
\end{aligned}
$$

where the second equality is from (2.3). For (2.5):

$$
\begin{aligned}
|\mathbf{x}-\mathbf{y}|^{2}= & |\mathbf{x}-\mathbf{c}|^{2}+|\mathbf{y}-\mathbf{c}|^{2}-2(\mathbf{x}-\mathbf{c}) \cdot(\mathbf{y}-\mathbf{c})= \\
= & \left(R^{2}-\frac{1}{2} \sum_{i=0}^{n} \sum_{j=0}^{n} d_{i j}^{2} x_{i} x_{j}\right)+\left(R^{2}-\frac{1}{2} \sum_{i=0}^{n} \sum_{j=0}^{n} d_{i j}^{2} y_{i} y_{j}\right)- \\
& \quad-\left(2 R^{2}-\frac{1}{2} \sum_{i=0}^{n} \sum_{j=0}^{n} d_{i j}^{2}\left(x_{i} y_{j}+y_{i} x_{j}\right)\right)= \\
= & -\frac{1}{2} \sum_{i=0}^{n} \sum_{j=0}^{n} d_{i j}^{2}\left(x_{i}-y_{i}\right)\left(x_{j}-y_{j}\right)
\end{aligned}
$$

where the second equality is from (2.4). For (2.6):

$$
\begin{aligned}
& \sum_{j=0}^{n}\left|\mathbf{t}_{j}\right|\left|\mathbf{q}_{j}-\mathbf{i}\right|^{2}=-\frac{1}{2} \sum_{j=0}^{n}\left|\mathbf{t}_{j}\right| \sum_{k=0}^{n} \sum_{\ell=0}^{n} d_{k \ell}^{2}\left(\delta_{k j}-r\left|\mathbf{t}_{k}\right|\right)\left(\delta_{\ell j}-r\left|\mathbf{t}_{\ell}\right|\right)= \\
& =\frac{1}{2} \sum_{j=0}^{n}\left|\mathbf{t}_{j}\right|\left(r \sum_{k=0}^{n} d_{k j}^{2}\left|\mathbf{t}_{k}\right|+r \sum_{\ell=0}^{n} d_{j \ell}^{2}\left|\mathbf{t}_{\ell}\right|-r^{2} \sum_{k=0}^{n} \sum_{\ell=0}^{n} d_{k \ell}^{2}\left|\mathbf{t}_{k}\right|\left|\mathbf{t}_{\ell}\right|\right)= \\
& =\sum_{j=0}^{n}\left|\mathbf{t}_{j}\right|\left(r \sum_{k=0}^{n} d_{k j}^{2}\left|\mathbf{t}_{k}\right|-\left(R^{2}-|\mathbf{c}-\mathbf{i}|^{2}\right)\right)= \\
& =\frac{2\left(R^{2}-|\mathbf{c}-\mathbf{i}|^{2}\right)}{r}-\frac{R^{2}-|\mathbf{c}-\mathbf{i}|^{2}}{r}=\frac{R^{2}-|\mathbf{c}-\mathbf{i}|^{2}}{r},
\end{aligned}
$$

where the first equality is from (2.5) for $\mathbf{x}=\mathbf{q}_{j}$ and $\mathbf{y}=\mathbf{i}$, and third and fourth equalities are from (2.4) for $\mathbf{x}=\mathbf{y}=\mathbf{i}$.

Remark 2.3. The square of the circumradius can be represented by the following (notice that this fraction is irreducible as a division of polynomials of $\mathbb{C}\left[\ldots, d_{i j}, \ldots\right]$, 
see $[3])$ :

$$
R^{2}=-\left(-\frac{1}{2}\right)^{n+1} \operatorname{det}\left(\begin{array}{ccc}
d_{00}^{2} & \cdots & d_{0 n}^{2} \\
\vdots & & \vdots \\
d_{n 0}^{2} & \cdots & d_{n n}^{2}
\end{array}\right) / \tilde{V}^{2} .
$$

It is from $\S 9.7 .3 .7$ of [1] or the following caluculation

$$
\begin{aligned}
& \operatorname{det}\left(\begin{array}{ccc}
d_{00}^{2} & \cdots & d_{0 n}^{2} \\
\vdots & & \vdots \\
d_{n 0}^{2} & \cdots & d_{n n}^{2}
\end{array}\right)=\operatorname{det}\left(\begin{array}{cccc}
d_{00}^{2} & \cdots & d_{0 n}^{2} & -1 \\
\vdots & & \vdots & \vdots \\
d_{n 0}^{2} & \cdots & d_{n n}^{2} & -1 \\
0 & \cdots & 0 & 1
\end{array}\right)= \\
& =\operatorname{det}\left(\begin{array}{cccc}
d_{00}^{2} & \cdots & d_{0 n}^{2} & -1 \\
\vdots & & \vdots & \vdots \\
d_{n 0}^{2} & \cdots & d_{n n}^{2} & -1 \\
\sum_{i=0}^{n} d_{i 0}^{2} c_{i} & \cdots & \sum_{i=0}^{n} d_{i n}^{2} c_{i} & 1-\sum_{i=0}^{n} c_{i}
\end{array}\right)= \\
& =\operatorname{det}\left(\begin{array}{cccc}
d_{00}^{2} & \cdots & d_{0 n}^{2} & -1 \\
\vdots & & \vdots & \vdots \\
d_{n 0}^{2} & \cdots & d_{n n}^{2} & -1 \\
2 R^{2} & \cdots & 2 R^{2} & 0
\end{array}\right)=-2 R^{2} \operatorname{det}\left(\begin{array}{cccc}
d_{00}^{2} & \cdots & d_{0 n}^{2} & 1 \\
\vdots & & \vdots & \vdots \\
d_{n 0}^{2} & \cdots & d_{n n}^{2} & 1 \\
1 & \cdots & 1 & 0
\end{array}\right)= \\
& =-(-2)^{n+1} R^{2} \tilde{V}^{2},
\end{aligned}
$$

where the third equality is from (2.3) and the last equality is from

$$
\begin{aligned}
& \left(\begin{array}{cccccc}
1 & 0 & \cdots & 0 & -1 & -d_{0 n}^{2} \\
0 & \ddots & \ddots & \vdots & \vdots & \vdots \\
\vdots & \ddots & \ddots & 0 & \vdots & \vdots \\
\vdots & & \ddots & \ddots & -1 & -d_{n-1, n}^{2} \\
\vdots & & & \ddots & \ddots & 0 \\
0 & \cdots & \cdots & \cdots & 0 & 1
\end{array}\right)\left(\begin{array}{cccc}
d_{00}^{2} & \cdots & d_{0 n}^{2} & 1 \\
\vdots & & \vdots & \vdots \\
d_{n 0}^{2} & \cdots & d_{n n}^{2} & 1 \\
1 & \cdots & 1 & 0
\end{array}\right)= \\
& =\left(\begin{array}{cccc}
d_{00}^{2}-d_{n 0}^{2}-d_{0 n}^{2} & \cdots & d_{0 n}^{2}-d_{n n}^{2}-d_{0 n}^{2} & 1-1 \\
\vdots & & \vdots & \vdots \\
d_{n-1,0}^{2}-d_{n 0}^{2}-d_{n-1, n}^{2} & \cdots & d_{n-1, n}^{2}-d_{n n}^{2}-d_{n-1, n}^{2} & 1-1 \\
d_{n 0}^{2} & \cdots & d_{n n}^{2} & 1 \\
1 & \cdots & 1 & 0
\end{array}\right)= \\
& =\left(\begin{array}{ccccc}
-2\left(\mathbf{q}_{0}-\mathbf{q}_{n}\right) \cdot\left(\mathbf{q}_{0}-\mathbf{q}_{n}\right) & \cdots & -2\left(\mathbf{q}_{0}-\mathbf{q}_{n}\right) \cdot\left(\mathbf{q}_{n-1}-\mathbf{q}_{n}\right) & 0 & 0 \\
\vdots & & \vdots & \vdots & \vdots \\
-2\left(\mathbf{q}_{n-1}-\mathbf{q}_{n}\right) \cdot\left(\mathbf{q}_{0}-\mathbf{q}_{n}\right) & \cdots & -2\left(\mathbf{q}_{n-1}-\mathbf{q}_{n}\right) \cdot\left(\mathbf{q}_{n-1}-\mathbf{q}_{n}\right) & 0 & 0 \\
d_{n 0}^{2} & \cdots & d_{n, n-1}^{2} & 0 & 1 \\
1 & \cdots & 1 & 1 & 0
\end{array}\right) .
\end{aligned}
$$


Let $n=2$. Then, it means $R=d_{12} d_{02} d_{01} /(2|\tilde{V}|)$. On the other hand, from Remark 2.2 ,

$$
\left|\mathbf{t}_{0}\right|=\frac{d_{12}}{|\tilde{V}|}, \quad\left|\mathbf{t}_{1}\right|=\frac{d_{02}}{|\tilde{V}|}, \quad\left|\mathbf{t}_{2}\right|=\frac{d_{01}}{|\tilde{V}|}, \quad \text { and } \quad r=\frac{|\tilde{V}|}{d_{12}+d_{02}+d_{01}}
$$

hold. These and (2.4) for $\mathbf{x}=\mathbf{y}=\mathbf{i}$ imply

$$
\begin{aligned}
& R^{2}-|\mathbf{c}-\mathbf{i}|^{2}=d_{12}^{2} r\left|\mathbf{t}_{1}\right| r\left|\mathbf{t}_{2}\right|+d_{02}^{2} r\left|\mathbf{t}_{0}\right| r\left|\mathbf{t}_{2}\right|+d_{01}^{2} r\left|\mathbf{t}_{0}\right| r\left|\mathbf{t}_{1}\right|= \\
& =d_{12}^{2} \frac{d_{02}}{d_{12}+d_{02}+d_{01}} \frac{d_{01}}{d_{12}+d_{02}+d_{01}}+d_{02}^{2} \frac{d_{12}}{d_{12}+d_{02}+d_{01}} \frac{d_{01}}{d_{12}+d_{02}+d_{01}}+ \\
& \quad+d_{01}^{2} \frac{d_{12}}{d_{12}+d_{02}+d_{01}} \frac{d_{02}}{d_{12}+d_{02}+d_{01}}=\frac{d_{12} d_{02} d_{01}}{d_{12}+d_{02}+d_{01}} .
\end{aligned}
$$

So we have

$$
\left(R^{2}-|\mathbf{c}-\mathbf{i}|^{2}\right) \frac{1}{r}=\frac{d_{12} d_{02} d_{01}}{d_{12}+d_{02}+d_{01}} \frac{d_{12}+d_{02}+d_{01}}{|\tilde{V}|}=2 R .
$$

\section{Escribed simplices and Generalization of Bevan Point Theorem.}

The following theorem is the explicit representation of the escribed simplex.

Lemma 3.1. Let $\Delta^{n}\left(\mathbf{q}_{0}^{\mathrm{E}}, \ldots, \mathbf{q}_{n}^{\mathrm{E}}\right)$ be the escribed simplex of $\Delta^{n}\left(\mathbf{q}_{0}, \ldots, \mathbf{q}_{n}\right)$. Then, we have

$$
\begin{gathered}
\mathbf{q}_{i}^{\mathrm{E}}=2 \mathbf{c}-\mathbf{i}-\frac{R^{2}-|\mathbf{c}-\mathbf{i}|^{2}}{r} \frac{\mathbf{t}_{i}}{\left|\mathbf{t}_{i}\right|}, \quad \mathbf{t}_{i}^{\mathrm{E}}=\frac{r\left|\mathbf{t}_{i}\right|\left(\mathbf{i}-\mathbf{q}_{i}\right)}{R^{2}-|\mathbf{c}-\mathbf{i}|^{2}}, \\
\mathbf{c}^{\mathrm{E}}=2 \mathbf{c}-\mathbf{i}, \quad R^{\mathrm{E}}=\frac{R^{2}-|\mathbf{c}-\mathbf{i}|^{2}}{r}, \quad c_{i}^{\mathrm{E}}=r\left|\mathbf{t}_{i}\right|, \\
\sum_{j=0}^{n} \frac{\left|\mathbf{t}_{j}^{\mathrm{E}}\right|^{2}}{c_{j}^{\mathrm{E}}}=\frac{1}{R^{2}-|\mathbf{c}-\mathbf{i}|^{2}}, \quad \sum_{j=0}^{n} \frac{\left|\mathbf{t}_{j}^{\mathrm{E}}\right|^{2}}{c_{j}^{\mathrm{E}}} \mathbf{q}_{j}^{\mathrm{E}}=\frac{\mathbf{i}}{R^{2}-|\mathbf{c}-\mathbf{i}|^{2}},
\end{gathered}
$$

for $i=0, \ldots, n$.

Proof. First, we have

$$
\begin{aligned}
\mathbf{q}_{i}^{\mathrm{E}} & =\mathbf{i}+\left(\mathbf{q}_{i}^{\mathrm{E}}-\mathbf{i}\right)=\mathbf{i}+\sum_{j=0}^{\substack{j \neq i \\
n}}\left(\left(\mathbf{q}_{i}^{\mathrm{E}}-\mathbf{i}\right) \cdot\left(\mathbf{q}_{j}-\mathbf{i}\right)\right)\left(\mathbf{t}_{j}-\frac{\left|\mathbf{t}_{j}\right|}{\left|\mathbf{t}_{i}\right|} \mathbf{t}_{i}\right)= \\
& =\mathbf{i}+\sum_{j=0}^{\substack{n \neq i \\
n}}\left|\mathbf{q}_{j}-\mathbf{i}\right|^{2}\left(\mathbf{t}_{j}-\frac{\left|\mathbf{t}_{j}\right|}{\left|\mathbf{t}_{i}\right|} \mathbf{t}_{i}\right)=\mathbf{i}+\sum_{j=0}^{n}\left|\mathbf{q}_{j}-\mathbf{i}\right|^{2}\left(\mathbf{t}_{j}-\frac{\left|\mathbf{t}_{j}\right|}{\left|\mathbf{t}_{i}\right|} \mathbf{t}_{i}\right)= \\
& =\mathbf{i}+\sum_{j=0}^{n}\left|\mathbf{q}_{j}-\mathbf{i}\right|^{2} \mathbf{t}_{j}-\sum_{j=0}^{n}\left|\mathbf{t}_{j}\right|\left|\mathbf{q}_{j}-\mathbf{i}\right|^{2} \frac{\mathbf{t}_{i}}{\left|\mathbf{t}_{i}\right|}= \\
& =\mathbf{i}-2(\mathbf{i}-\mathbf{c})-\frac{R^{2}-|\mathbf{c}-\mathbf{i}|^{2}}{r} \frac{\mathbf{t}_{i}}{\left|\mathbf{t}_{i}\right|},
\end{aligned}
$$

where the second equality is from the duality of $\left\{\mathbf{q}_{0}-\mathbf{i}, \ldots \widehat{\mathbf{q}_{i}-\mathbf{i}} \ldots, \mathbf{q}_{n}-\mathbf{i}\right\}$ and $\left\{\mathbf{t}_{0}-\frac{\left|\mathbf{t}_{0}\right|}{\left|\mathbf{t}_{i}\right|} \mathbf{t}_{i}, \ldots \mathbf{t}_{i}-\widehat{\frac{\left|\mathbf{t}_{i}\right|}{\left|\mathbf{t}_{i}\right|}} \mathbf{t}_{i} \ldots, \mathbf{t}_{n}-\frac{\left|\mathbf{t}_{n}\right|}{\left|\mathbf{t}_{i}\right|} \mathbf{t}_{i}\right\}$ (this is the special case of the duality of $\left\{\mathbf{q}_{0}-\mathbf{x}, \ldots \widehat{\mathbf{q}_{i}-\mathbf{x}} \ldots, \mathbf{q}_{n}-\mathbf{x}\right\}$ and $\left.\left\{\mathbf{t}_{0}-\frac{x_{0}}{x_{i}} \mathbf{t}_{i}, \ldots \widehat{\mathbf{t}_{i}-\frac{x_{i}}{x_{i}}} \mathbf{t}_{i} \ldots, \mathbf{t}_{n}-\frac{x_{n}}{x_{i}} \mathbf{t}_{i}\right\}\right)$, the third equality is from the perpendicularity of vectors $\mathbf{q}_{j}-\mathbf{i}$ and $\mathbf{q}_{i}^{\mathrm{E}}-\mathbf{q}_{j}$ for $j$ distinct 
from $i$, and the last equality is from (2.1) for $\mathbf{x}=\mathbf{i}$ and (2.6). From the calculation above, the following equations are obvious:

$$
\mathbf{c}^{\mathrm{E}}=2 \mathbf{c}-\mathbf{i}, \quad R^{\mathrm{E}}=\frac{R^{2}-|\mathbf{c}-\mathbf{i}|^{2}}{r} .
$$

Secondly, we can represent the vector of the dual basis:

$$
\mathbf{t}_{i}^{\mathrm{E}}=\frac{r\left|\mathbf{t}_{i}\right|\left(\mathbf{i}-\mathbf{q}_{i}\right)}{R^{2}-|\mathbf{c}-\mathbf{i}|^{2}}
$$

from the calculation

$$
\begin{aligned}
\left(\mathbf{q}_{k}^{\mathrm{E}}-\mathbf{q}_{\ell}^{\mathrm{E}}\right) \cdot \frac{r\left|\mathbf{t}_{i}\right|\left(\mathbf{i}-\mathbf{q}_{i}\right)}{R^{2}-|\mathbf{c}-\mathbf{i}|^{2}} & =\frac{R^{2}-|\mathbf{c}-\mathbf{i}|^{2}}{r}\left(-\frac{\mathbf{t}_{k}}{\left|\mathbf{t}_{k}\right|}+\frac{\mathbf{t}_{\ell}}{\left|\mathbf{t}_{\ell}\right|}\right) \cdot \frac{r\left|\mathbf{t}_{i}\right|\left(\mathbf{i}-\mathbf{q}_{i}\right)}{R^{2}-|\mathbf{c}-\mathbf{i}|^{2}}= \\
& =\left(-\frac{\mathbf{t}_{k}}{\left|\mathbf{t}_{k}\right|}+\frac{\mathbf{t}_{\ell}}{\left|\mathbf{t}_{\ell}\right|}\right) \cdot\left|\mathbf{t}_{i}\right| \sum_{j=0}^{n} r\left|\mathbf{t}_{j}\right|\left(\mathbf{q}_{j}-\mathbf{q}_{i}\right)= \\
& =\left|\mathbf{t}_{i}\right| \sum_{j=0}^{n} r\left|\mathbf{t}_{j}\right|\left(-\frac{\delta_{k j}-\delta_{k i}}{\left|\mathbf{t}_{k}\right|}+\frac{\delta_{\ell j}-\delta_{\ell i}}{\left|\mathbf{t}_{\ell}\right|}\right)= \\
& =\left|\mathbf{t}_{i}\right|\left(-\frac{r\left|\mathbf{t}_{k}\right|-\delta_{k i}}{\left|\mathbf{t}_{k}\right|}+\frac{r\left|\mathbf{t}_{\ell}\right|-\delta_{\ell i}}{\left|\mathbf{t}_{\ell}\right|}\right)=\delta_{k i}-\delta_{\ell i},
\end{aligned}
$$

for $k, \ell=0, \ldots, n$. Thirdly, we have

$$
\begin{aligned}
c_{i}^{\mathrm{E}}-\delta_{n i} & =\left(\mathbf{c}^{\mathrm{E}}-\mathbf{q}_{n}^{\mathrm{E}}\right) \cdot \mathbf{t}_{i}^{\mathrm{E}}=\frac{R^{2}-|\mathbf{c}-\mathbf{i}|^{2}}{r} \frac{\mathbf{t}_{n}}{\left|\mathbf{t}_{n}\right|} \cdot \frac{r\left|\mathbf{t}_{i}\right|\left(\mathbf{i}-\mathbf{q}_{i}\right)}{R^{2}-|\mathbf{c}-\mathbf{i}|^{2}}= \\
& =\frac{\mathbf{t}_{n}}{\left|\mathbf{t}_{n}\right|} \cdot\left|\mathbf{t}_{i}\right| \sum_{j=0}^{n} r\left|\mathbf{t}_{j}\right|\left(\mathbf{q}_{j}-\mathbf{q}_{i}\right)=\frac{\left|\mathbf{t}_{i}\right|}{\left|\mathbf{t}_{n}\right|} \sum_{j=0}^{n} r\left|\mathbf{t}_{j}\right|\left(\delta_{n j}-\delta_{n i}\right)= \\
& =\frac{\left|\mathbf{t}_{i}\right|}{\left|\mathbf{t}_{n}\right|}\left(r\left|\mathbf{t}_{n}\right|-\delta_{n i}\right)=r\left|\mathbf{t}_{i}\right|-\delta_{n i} .
\end{aligned}
$$

Fourthly, the results above imply

$$
\sum_{j=0}^{n} \frac{\left|\mathbf{t}_{j}^{\mathrm{E}}\right|^{2}}{c_{j}^{\mathrm{E}}}=\frac{r}{\left(R^{2}-|\mathbf{c}-\mathbf{i}|^{2}\right)^{2}} \sum_{j=0}^{n}\left|\mathbf{t}_{j}\right|\left|\mathbf{i}-\mathbf{q}_{j}\right|^{2}=\frac{1}{R^{2}-|\mathbf{c}-\mathbf{i}|^{2}},
$$

where the last equality is from (2.6). At last, we have

$$
\begin{aligned}
\sum_{j=0}^{n} \frac{\left|\mathbf{t}_{j}^{\mathrm{E}}\right|^{2}}{c_{j}^{\mathrm{E}}} \mathbf{q}_{j}^{\mathrm{E}} & =\frac{r}{\left(R^{2}-|\mathbf{c}-\mathbf{i}|^{2}\right)^{2}} \sum_{j=0}^{n}\left|\mathbf{t}_{j}\right|\left|\mathbf{i}-\mathbf{q}_{j}\right|^{2}\left((2 \mathbf{c}-\mathbf{i})-\frac{R^{2}-|\mathbf{c}-\mathbf{i}|^{2}}{r} \frac{\mathbf{t}_{j}}{\left|\mathbf{t}_{j}\right|}\right)= \\
& =\frac{1}{R^{2}-|\mathbf{c}-\mathbf{i}|^{2}}\left((2 \mathbf{c}-\mathbf{i})-\sum_{j=0}^{n}\left|\mathbf{i}-\mathbf{q}_{j}\right|^{2} \mathbf{t}_{j}\right)= \\
& =\frac{1}{R^{2}-|\mathbf{c}-\mathbf{i}|^{2}}((2 \mathbf{c}-\mathbf{i})+2(\mathbf{i}-\mathbf{c}))=\frac{\mathbf{i}}{R^{2}-|\mathbf{c}-\mathbf{i}|^{2}},
\end{aligned}
$$

where the second equality is from (2.6) and the third equality is from (2.1) for $\mathbf{x}=\mathbf{i}$, respectively.

Remark 3.1. Notice that $c_{i}^{\mathrm{E}}=r\left|\mathbf{t}_{i}\right|>0$ for $i=0, \ldots, n$, so the circumcenter of $\Delta^{n}\left(\mathbf{q}_{0}^{\mathrm{E}}, \ldots, \mathbf{q}_{n}^{\mathrm{E}}\right)$ is an interior of it. 
The lemma above is essentially including generalized Bevan point theorem.

Proof of Theorem 1.2. We already get two equations $\mathbf{c}^{\mathrm{E}}=2 \mathbf{c}-\mathbf{i}$ and $R^{\mathrm{E}}=\frac{R^{2}-|\mathbf{c}-\mathbf{i}|^{2}}{r}$. Moreover, the vector $\mathbf{c}^{\mathrm{E}}-\mathbf{q}_{i}^{\mathrm{E}}$ is parallel to $\mathbf{t}_{i}$, so it is perpendicular to the face $\Delta^{n-1}\left(\mathbf{q}_{0}, \ldots \widehat{\mathbf{q}_{i}} \ldots, \mathbf{q}_{n}\right)$.

\section{INSCRIBED SIMPLICES.}

The following lemma and theorem give us the explicit representation of the inscribed simplex.

Lemma 4.1. For a simplex $\Delta^{n}\left(\mathbf{q}_{0}, \ldots, \mathbf{q}_{n}\right)$ whose circumcenter is an interior of it (i.e., $c_{j}>0$ for $\left.j=0, \ldots, n\right)$, let $\Delta^{n}\left(\mathbf{q}_{0}^{\mathrm{I}}, \ldots, \mathbf{q}_{n}^{\mathrm{I}}\right)$ be the simplex with vertices

$$
\mathbf{q}_{i}^{\mathrm{I}}=\frac{1}{\sum_{j=0}^{n} \frac{\left|\mathbf{t}_{j}\right|^{2}}{c_{j}}}\left(\sum_{j=0}^{n} \frac{\left|\mathbf{t}_{j}\right|^{2}}{c_{j}} \mathbf{q}_{j}-\frac{\mathbf{t}_{i}}{c_{i}}\right) .
$$

Then, we have the following equations:

$$
\begin{gathered}
\mathbf{t}_{i}^{\mathrm{I}}=\left(\sum_{j=0}^{n} \frac{\left|\mathbf{t}_{j}\right|^{2}}{c_{j}}\right) c_{i}\left(\mathbf{c}-\mathbf{q}_{i}\right), \quad\left|\mathbf{t}_{i}^{\mathrm{I}}\right|=R\left(\sum_{j=0}^{n} \frac{\left|\mathbf{t}_{j}\right|^{2}}{c_{j}}\right) c_{i}, \\
r^{\mathrm{I}}=\frac{1}{R \sum_{j=0}^{n} \frac{\left|\mathbf{t}_{j}\right|^{2}}{c_{j}}}, \quad \mathbf{i}^{\mathrm{I}}=\frac{1}{\sum_{j=0}^{n} \frac{\left|\mathbf{t}_{j}\right|^{2}}{c_{j}}} \sum_{j=0}^{n} \frac{\left|\mathbf{t}_{j}\right|^{2}}{c_{j}} \mathbf{q}_{j}, \\
2 \mathbf{c}^{\mathrm{I}}-\mathbf{i}^{\mathrm{I}}=\mathbf{c}, \quad\left(R^{\mathrm{I}}\right)^{2}-\left|\mathbf{c}^{\mathrm{I}}-\mathbf{i}^{\mathrm{I}}\right|^{2}=\frac{1}{\sum_{j=0}^{n} \frac{\left|\mathbf{t}_{j}\right|^{2}}{c_{j}}},
\end{gathered}
$$

for $i=0, \ldots, n$.

Proof. The equation

$$
\mathbf{t}_{i}^{\mathrm{I}}=\left(\sum_{j=0}^{n} \frac{\left|\mathbf{t}_{j}\right|^{2}}{c_{j}}\right) c_{i}\left(\mathbf{c}-\mathbf{q}_{i}\right)
$$

is implied from

$$
\begin{aligned}
& \left(\mathbf{q}_{k}^{\mathrm{I}}-\mathbf{q}_{\ell}^{\mathrm{I}}\right) \cdot\left(\sum_{j=0}^{n} \frac{\left|\mathbf{t}_{j}\right|^{2}}{c_{j}}\right) c_{i}\left(\mathbf{c}-\mathbf{q}_{i}\right)=\frac{-\frac{\mathbf{t}_{k}}{c_{k}}+\frac{\mathbf{t}_{\ell}}{c_{\ell}}}{\sum_{j=0}^{n} \frac{\left|\mathbf{t}_{j}\right|^{2}}{c_{j}}} \cdot\left(\sum_{j=0}^{n} \frac{\left|\mathbf{t}_{j}\right|^{2}}{c_{j}}\right) c_{i}\left(\mathbf{c}-\mathbf{q}_{i}\right)= \\
& =\left(-\frac{\mathbf{t}_{k}}{c_{k}}+\frac{\mathbf{t}_{\ell}}{c_{\ell}}\right) \cdot c_{i}\left(\mathbf{c}-\mathbf{q}_{i}\right)=\left(-\frac{\mathbf{t}_{k}}{c_{k}}+\frac{\mathbf{t}_{\ell}}{c_{\ell}}\right) \cdot c_{i} \sum_{j=0}^{n} c_{j}\left(\mathbf{q}_{j}-\mathbf{q}_{i}\right)= \\
& =c_{i} \sum_{j=0}^{n} c_{j}\left(-\frac{\delta_{k j}-\delta_{k i}}{c_{k}}+\frac{\delta_{\ell j}-\delta_{\ell i}}{c_{\ell}}\right)=c_{i}\left(-\frac{c_{k}-\delta_{k i}}{c_{k}}+\frac{c_{\ell}-\delta_{\ell i}}{c_{\ell}}\right)=\delta_{k i}-\delta_{\ell i},
\end{aligned}
$$

for $k, \ell=0, \ldots, n$. So, it is obvious that

$$
\left|\mathbf{t}_{i}^{\mathrm{I}}\right|=R\left(\sum_{j=0}^{n} \frac{\left|\mathbf{t}_{j}\right|^{2}}{c_{j}}\right) c_{i} .
$$

Hence, we have

$$
\frac{1}{r^{\mathrm{I}}}=\sum_{i=0}^{n}\left|\mathbf{t}_{i}^{\mathrm{I}}\right|=R\left(\sum_{j=0}^{n} \frac{\left|\mathbf{t}_{j}\right|^{2}}{c_{j}}\right) \sum_{i=0}^{n} c_{i}=R \sum_{j=0}^{n} \frac{\left|\mathbf{t}_{j}\right|^{2}}{c_{j}} .
$$


We also have

$$
\begin{aligned}
\mathbf{i}^{\mathrm{I}} & =\sum_{i=0}^{n} r^{\mathrm{I}}\left|\mathbf{t}_{i}^{\mathrm{I}}\right| \mathbf{q}_{i}^{\mathrm{I}}=\sum_{i=0}^{n} c_{i} \cdot \frac{1}{\sum_{j=0}^{n} \frac{\left|\mathbf{t}_{j}\right|^{2}}{c_{j}}}\left(\sum_{j=0}^{n} \frac{\left|\mathbf{t}_{j}\right|^{2}}{c_{j}} \mathbf{q}_{j}-\frac{\mathbf{t}_{i}}{c_{i}}\right)= \\
& =\frac{1}{\sum_{j=0}^{n} \frac{\left|\mathbf{t}_{j}\right|^{2}}{c_{j}}} \sum_{j=0}^{n} \frac{\left|\mathbf{t}_{j}\right|^{2}}{c_{j}} \mathbf{q}_{j}-\frac{\sum_{i=0}^{n} \mathbf{t}_{i}}{\sum_{j=0}^{n} \frac{\left|\mathbf{t}_{j}\right|^{2}}{c_{j}}}=\frac{1}{\sum_{j=0}^{n} \frac{\left|\mathbf{t}_{j}\right|^{2}}{c_{j}}} \sum_{j=0}^{n} \frac{\left|\mathbf{t}_{j}\right|^{2}}{c_{j}} \mathbf{q}_{j} .
\end{aligned}
$$

The result of the calculation

$$
\begin{aligned}
& \left|\frac{\mathbf{c}+\mathbf{i}^{\mathrm{I}}}{2}-\mathbf{q}_{k}^{\mathrm{I}}\right|^{2}-\left|\frac{\mathbf{c}+\mathbf{i}^{\mathrm{I}}}{2}-\mathbf{i}^{\mathrm{I}}\right|^{2}=\left(\mathbf{i}^{\mathrm{I}}-\mathbf{q}_{k}^{\mathrm{I}}\right) \cdot\left(\mathbf{c}-\mathbf{q}_{k}^{\mathrm{I}}\right)= \\
& =\frac{\mathbf{t}_{k}}{c_{k} \sum_{j=0}^{n} \frac{\left|\mathbf{t}_{j}\right|^{2}}{c_{j}}} \cdot\left(\left(\mathbf{c}-\mathbf{q}_{n}\right)-\left(\mathbf{q}_{k}^{\mathrm{I}}-\mathbf{q}_{n}\right)\right)= \\
& =\frac{\mathbf{t}_{k}}{c_{k} \sum_{j=0}^{n} \frac{\left|\mathbf{t}_{j}\right|^{2}}{c_{j}}} \cdot\left(\sum_{j=0}^{n} c_{j}\left(\mathbf{q}_{j}-\mathbf{q}_{n}\right)-\frac{1}{\sum_{j=0}^{n} \frac{\left|\mathbf{t}_{j}\right|^{2}}{c_{j}}}\left(\sum_{j=0}^{n} \frac{\left|\mathbf{t}_{j}\right|^{2}}{c_{j}}\left(\mathbf{q}_{j}-\mathbf{q}_{n}\right)-\frac{\mathbf{t}_{k}}{c_{k}}\right)\right)= \\
& =\frac{1}{c_{k} \sum_{j=0}^{n} \frac{\left|\mathbf{t}_{j}\right|^{2}}{c_{j}}}\left(\sum_{j=0}^{n} c_{j}\left(\delta_{k j}-\delta_{k n}\right)-\frac{1}{\sum_{j=0}^{n} \frac{\left|\mathbf{t}_{j}\right|^{2}}{c_{j}}}\left(\sum_{j=0}^{n} \frac{\left|\mathbf{t}_{j}\right|^{2}}{c_{j}}\left(\delta_{k j}-\delta_{k n}\right)-\frac{\left|\mathbf{t}_{k}\right|^{2}}{c_{k}}\right)\right)= \\
& =\frac{1}{c_{k} \sum_{j=0}^{n} \frac{\left|\mathbf{t}_{j}\right|^{2}}{c_{j}}}\left(\left(c_{k}-\delta_{k n}\right)-\frac{1}{\sum_{j=0}^{n} \frac{\left|\mathbf{t}_{j}\right|^{2}}{c_{j}}}\left(\left(\frac{\left|\mathbf{t}_{k}\right|^{2}}{c_{k}}-\delta_{k n} \sum_{j=0}^{n} \frac{\left|\mathbf{t}_{j}\right|^{2}}{c_{j}}\right)-\frac{\left|\mathbf{t}_{k}\right|^{2}}{c_{k}}\right)\right)= \\
& =\frac{1}{\sum_{j=0}^{n} \frac{\left|\mathbf{t}_{j}\right|^{2}}{c_{j}}}
\end{aligned}
$$

does not depend on the index $k=0, \ldots, n$, so we have

$$
\mathbf{c}^{\mathrm{I}}=\frac{\mathbf{c}+\mathbf{i}^{\mathrm{I}}}{2}, \quad \text { and } \quad\left(R^{\mathrm{I}}\right)^{2}-\left|\frac{\mathbf{c}+\mathbf{i}^{\mathrm{I}}}{2}-\mathbf{i}^{\mathrm{I}}\right|^{2}=\frac{1}{\sum_{j=0}^{n} \frac{\left|\mathbf{t}_{j}\right|^{2}}{c_{j}}} .
$$

Theorem 4.1. $\Delta^{n}\left(\mathbf{q}_{0}^{\mathrm{I}}, \ldots, \mathbf{q}_{n}^{\mathrm{I}}\right)$ is the inscribed simplex of $\Delta^{n}\left(\mathbf{q}_{0}, \ldots, \mathbf{q}_{n}\right)$, i.e., for arbitrary $\Delta^{n}\left(\mathbf{q}_{0}, \ldots, \mathbf{q}_{n}\right)$, the simplex $\Delta^{n}\left(\left(\mathbf{q}^{\mathrm{E}}\right)_{0}^{\mathrm{I}}, \ldots,\left(\mathbf{q}^{\mathrm{E}}\right)_{n}^{\mathrm{I}}\right)$ is consistent with $\Delta^{n}\left(\mathbf{q}_{0}, \ldots, \mathbf{q}_{n}\right)$, and for arbitrary $\Delta^{n}\left(\mathbf{q}_{0}, \ldots, \mathbf{q}_{n}\right)$ whose circumcenter is an interior of it, the simplex $\Delta^{n}\left(\left(\mathbf{q}^{\mathrm{I}}\right)_{0}^{\mathrm{E}}, \ldots,\left(\mathbf{q}^{\mathrm{I}}\right)_{n}^{\mathrm{E}}\right)$ is consistent with $\Delta^{n}\left(\mathbf{q}_{0}, \ldots, \mathbf{q}_{n}\right)$.

Proof. We can calculate the vertices of the simplices directly:

$$
\begin{aligned}
\left(\mathbf{q}^{\mathrm{E}}\right)_{i}^{\mathrm{I}} & =\frac{1}{\sum_{j=0}^{n} \frac{\left|\mathbf{t}_{j}^{\mathrm{E}}\right|^{2}}{c_{j}^{\mathrm{E}}}}\left(\sum_{j=0}^{n} \frac{\left|\mathbf{t}_{j}^{\mathrm{E}}\right|^{2}}{c_{j}^{\mathrm{E}}} \mathbf{q}_{j}^{\mathrm{E}}-\frac{\mathbf{t}_{i}^{\mathrm{E}}}{c_{i}^{\mathrm{E}}}\right)= \\
& =\left(R^{2}-|\mathbf{c}-\mathbf{i}|^{2}\right)\left(\frac{\mathbf{i}}{R^{2}-|\mathbf{c}-\mathbf{i}|^{2}}-\frac{\mathbf{i}-\mathbf{q}_{i}}{R^{2}-|\mathbf{c}-\mathbf{i}|^{2}}\right)=\mathbf{q}_{i}, \\
\left(\mathbf{q}^{\mathrm{I}}\right)_{i}^{\mathrm{E}} & =\left(2 \mathbf{c}^{\mathrm{I}}-\mathbf{i}^{\mathrm{I}}\right)-\frac{\left(R^{\mathrm{I}}\right)^{2}-\left|\mathbf{c}^{\mathrm{I}}-\mathbf{i}^{\mathrm{I}}\right|^{2}}{r^{\mathrm{I}}} \frac{\mathbf{t}_{i}^{\mathrm{I}}}{\left|\mathbf{t}_{i}^{\mathrm{I}}\right|}=\mathbf{c}-R \frac{\mathbf{c}-\mathbf{q}_{i}}{R}=\mathbf{q}_{i} .
\end{aligned}
$$


Remark 4.1. We can consider other types of correspondence. For example, for $\varepsilon_{0}=\cdots=\varepsilon_{n-2}=1$ and $\varepsilon_{n-1}=\varepsilon_{n}=-1$,

$$
\mathbf{i}_{n-1, n}=r_{n-1, n} \sum_{j=0}^{n} \varepsilon_{j}\left|\mathbf{t}_{j}\right| \mathbf{q}_{j} \quad \text { and } \quad r_{n-1, n}=\frac{1}{\sum_{j=0}^{n} \varepsilon_{j}\left|\mathbf{t}_{j}\right|}
$$

are the center and the radius of one of spheres touching all affined spaces spanned by $(n-1)$-dimensional faces of a simplex $\Delta^{n}\left(\mathbf{q}_{0}, \ldots, \mathbf{q}_{n}\right)$. For $\Delta^{n}\left(\mathbf{q}_{0}, \ldots, \mathbf{q}_{n}\right)$ with $R^{2}-\left|\mathbf{c}-\mathbf{i}_{n-1, n}\right|^{2} \neq 0$ and $\sum_{j=0}^{n} \varepsilon_{j}\left|\mathbf{t}_{j}\right|>0$, let

$$
\mathbf{q}_{i}^{\mathrm{E}_{n-1, n}}=2 \mathbf{c}-\mathbf{i}_{n-1, n}-\frac{R^{2}-\left|\mathbf{c}-\mathbf{i}_{n-1, n}\right|^{2}}{r_{n-1, n}} \frac{\mathbf{t}_{i}}{\varepsilon_{i}\left|\mathbf{t}_{i}\right|},
$$

for $i=0, \ldots, n$. Then, $\Delta^{n}\left(\mathbf{q}_{0}^{\mathrm{E}_{n-1, n}}, \ldots, \mathbf{q}_{n}^{\mathrm{E}_{n-1, n}}\right)$ is the simplex with $\sum_{j=0}^{n} \frac{\left|\mathbf{t}_{j}^{\mathrm{E}_{n-1, n}}\right|^{2}}{c_{j}^{\mathrm{E}_{n-1, n}}} \neq$ 0 and $\varepsilon_{i} c_{i}^{\mathrm{E}_{n-1, n}}>0$ for $i=0, \ldots, n$ (in particular, $\varepsilon_{i} c_{i}^{\mathrm{E}_{n-1, n}}=r_{n-1, n}\left|\mathbf{t}_{i}\right|>0$ ). On the other hand, for a simplex $\Delta^{n}\left(\mathbf{q}_{0}, \ldots, \mathbf{q}_{n}\right)$ with $\sum_{j=0}^{n} \frac{\left|\mathbf{t}_{j}\right|^{2}}{c_{j}} \neq 0$ and $\varepsilon_{i} c_{i}>0$ for $i=0, \ldots, n$, let

$$
\mathbf{q}_{i}^{\mathrm{I}_{n-1, n}}=\frac{1}{\sum_{j=0}^{n} \frac{\left|\mathbf{t}_{j}\right|^{2}}{c_{j}}}\left(\sum_{j=0}^{n} \frac{\left|\mathbf{t}_{j}\right|^{2}}{c_{j}} \mathbf{q}_{j}-\frac{\mathbf{t}_{i}}{c_{i}}\right)
$$

for $i=0, \ldots, n$ (notice that the right-hand side above is same to the right-hand side of the formula of the inscribed simplex). Then, $\Delta^{n}\left(\mathbf{q}_{0}^{\mathbf{I}_{n-1, n}}, \ldots, \mathbf{q}_{n-1, n}\right)$ is the simplex with $\left(R^{\mathrm{I}_{n-1, n}}\right)^{2}-\left|\mathbf{c}^{\mathrm{I}_{n-1, n}}-\mathbf{i}_{n-1, n}^{\mathrm{I}_{n-1, n}}\right|^{2} \neq 0$ and $\sum_{j=0}^{n} \varepsilon_{j}\left|\mathbf{t}_{j}^{\mathrm{I}_{n-1, n}}\right|>0$ (in particular, $\left.\sum_{j=0}^{n} \varepsilon_{j}\left|\mathbf{t}_{j}^{\mathrm{I}_{n-1, n}}\right|=\sum_{j=0}^{n} R\left|\sum_{k=0}^{n} \frac{\left|\mathbf{t}_{k}\right|^{2}}{c_{k}}\right| c_{j}=R\left|\sum_{k=0}^{n} \frac{\left|\mathbf{t}_{k}\right|^{2}}{c_{k}}\right|>0\right)$. Similar to the theorem above, these mapppings $\Delta^{n}\left(\mathbf{q}_{0}, \ldots, \mathbf{q}_{n}\right) \mapsto \Delta^{n}\left(\mathbf{q}_{0}^{\mathrm{E}_{n-1, n}}, \ldots, \mathbf{q}_{n}^{\mathrm{E}_{n-1, n}}\right)$ and $\Delta^{n}\left(\mathbf{q}_{0}, \ldots, \mathbf{q}_{n}\right) \mapsto \Delta^{n}\left(\mathbf{q}_{0}^{\mathrm{I}_{n-1, n}}, \ldots, \mathbf{q}_{n}^{\mathrm{I}_{n-1, n}}\right)$ are inverse mappings each other.

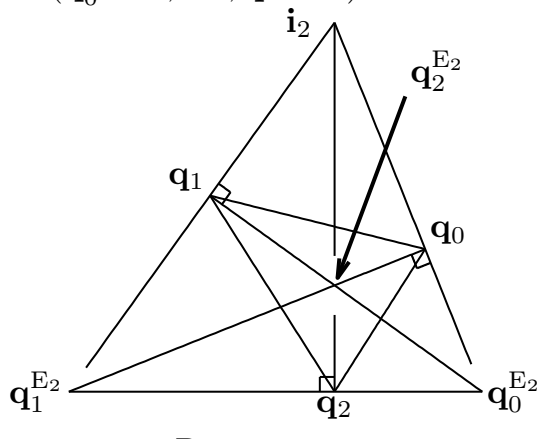

REFERENCES

[1] Berger, M., Geometry, Vol. 1, Springer-Verlag, Berlin, 1987.

[2] Coxeter, H. S. M. and Greitzer, S. L., Geometry revisited, The Mathematical Association of America, Washington, 1967.

[3] D'Andrea, C. and Sombra, M., The Cayley-Menger determinant is irreducible for $n \geqq 3$ Siberian Math. J. 46(2005), 71-76.

[4] Satô, K., Spherical simplices and their polars, Quart. J. Math. 58(2007), 107-126.

Laboratory of Mathematics, Faculty of Engineering, Tamagawa University, 6-1-1, Tamagawa-Gakuen, Machida, TOKYo 194-8610, JaPan

E-mail address: kenzi@eng.tamagawa.ac.jp 